Homology, Homotopy and Applications, vol.10(3), 2008, pp.27-43

\title{
GALOIS EXTENSIONS OF LUBIN-TATE SPECTRA
}

\author{
ANDREW BAKER AND BIRGIT RICHTER
}

(communicated by J. Michael Boardman)

Abstract

Let $E_{n}$ be the $n$-th Lubin-Tate spectrum at a prime $p$. There is a commutative $S$-algebra $E_{n}^{\mathrm{nr}}$ whose coefficients are built from the coefficients of $E_{n}$ and contain all roots of unity whose order is not divisible by $p$. For odd primes $p$ we show that $E_{n}^{\mathrm{nr}}$ does not have any non-trivial connected finite Galois extensions and is thus separably closed in the sense of Rognes. At the prime 2 we prove that there are no non-trivial connected Galois extensions of $E_{n}^{\mathrm{nr}}$ with Galois group a finite group $G$ with cyclic quotient. Our results carry over to the $K(n)$-local context.

\section{Introduction}

For a prime $p$, let $E_{n}$ be the Lubin-Tate spectrum whose coefficient ring is

$$
\pi_{*}\left(E_{n}\right)=W \mathbb{F}_{p^{n}}\left[\left[u_{1}, \ldots, u_{n-1}\right]\right]\left[u^{ \pm 1}\right],
$$

where $u$ is an element of degree -2 and the $u_{i}$ have degree zero. For a perfect field $\mathbb{k}$, $W \mathbb{k}$ denotes the ring of Witt vectors of $\mathbb{k}$. The ring $W \mathbb{F}_{p^{n}}\left[\left[u_{1}, \ldots, u_{n-1}\right]\right]$ represents deformations of the height $n$ Honda formal group law over $\mathbb{F}_{p^{n}}$. The spectrum $E_{2}$ features prominently in the work of Goerss, Henn, Mahowald and Rezk [GHMR] on the calculation of the homotopy groups of the $K(2)$-local sphere. Goerss, Hopkins and Miller $[\mathbf{R e}, \mathbf{G H}]$ establish an action of the extended Morava stabilizer group $\mathbb{G}_{n}$ by $E_{\infty}$-maps on $E_{n}$. Work by Devinatz and Hopkins $[\mathbf{D H}]$ on homotopy fixed point spectra identifies the $K(n)$-local sphere spectrum $L_{K(n)} S$ as the homotopy fixed points of the action of $\mathbb{G}_{n}$ on $E_{n}$ and Rognes [R, Section 5.4.1] interprets the map

$$
L_{K(n)} S \simeq E_{n}^{h \mathbb{G}_{n}} \longrightarrow E_{n}
$$

as a $K(n)$-local Galois extension with Galois group $\mathbb{G}_{n}$.

As observed in [R, Section 5.4.1], there is a $K(n)$-local Galois extension $E_{n} \longrightarrow$ $E_{n}^{\mathrm{nr}}$ obtained by adjoining all roots of unity of order prime to $p$ and then suitably completing the result, so that

$$
\pi_{*}\left(E_{n}^{\mathrm{nr}}\right)=W \overline{\mathbb{F}}_{p}\left[\left[u_{1}, \ldots, u_{n-1}\right]\right]\left[u^{ \pm 1}\right],
$$

where $\overline{\mathbb{F}}_{p}$ is the algebraic closure of $\mathbb{F}_{p^{n}}$. See Section 2 for more details on $E_{n}^{\mathrm{nr}}$.

Received December 6, 2007, revised July 11, 2008; published on December 5, 2008. 2000 Mathematics Subject Classification: 55P43, 13B05, 13K05; 55P60, 55N22.

Key words and phrases: Galois extensions, separable closure, Witt vectors, Lubin-Tate spectra.

This article is available at http://intlpress.com/HHA/v10/n3/a3

Copyright (c) 2008, International Press. Permission to copy for private use granted. 
Usually $E_{n}^{\mathrm{nr}}$ is thought of as the maximal (abelian) unramified extension of $E_{n}$ and our goal is to investigate the extent to which it deserves this name. The coefficients do not allow for non-trivial connected Galois extensions of graded commutative rings, and we will show that there are no non-trivial connected finite Galois extensions of $E_{n}^{\mathrm{nr}}$ as a commutative $S$-algebra, at least if we work away from the prime 2 . Here, we use the notion of connectedness in the sense of Rognes [R, 10.2], thus a connected commutative $S$-algebra is one without non-trivial idempotents. This is crucial because for every commutative $S$-algebra $A$ we can always consider the trivial $G$-Galois extension $A \longrightarrow \prod_{G} A$ for an arbitrary finite group $G$. We will recall some basic facts about connectedness in Section 3.

Our main result confirms Rognes' [R, Conjecture 1.4].

Theorem 1.1. For an odd prime $p$, let $B / E_{n}^{\mathrm{nr}}$ be a finite Galois extension with nontrivial Galois group. Then $B$ is not connected. Hence $E_{n}^{\mathrm{nr}}$ is a maximal connected Galois extension of $E_{n}$.

For $p=2$ we show that any finite Galois extension $B / E_{n}^{\mathrm{nr}}$ whose Galois group has a cyclic quotient is not connected. At the moment we are unable to prove that there are no non-trivial connected Galois extensions of $E_{n}^{\text {nr }}$ at $p=2$ with a Galois group which has only finite simple non-abelian quotients.

This result extends our earlier work of [BR:2, Example 42], in which we showed that each abelian Galois extension $E_{n}^{\mathrm{nr}} \longrightarrow B$ with Galois group whose order is prime to $p$ gives rise to an algebraic Galois extension $\pi_{*}\left(E_{n}^{\mathrm{nr}}\right) \longrightarrow \pi_{*}(B)$, where the target is concentrated in even degrees.

Rognes [R, Definition 10.3.1] calls a connected commutative $S$-algebra $A$ separably closed if there are no $G$-Galois extensions $A \longrightarrow B$ with $G$ finite and non-trivial and $B$ connected, i.e., if each finite $G$-Galois extension $A \longrightarrow B$ has a trivial Galois group or $B$ not connected.

In this terminology we prove that for an odd prime $p$ the spectrum $E_{n}^{\mathrm{nr}}$ is separably closed. We conjecture that it is also separably closed when $p=2$.

In Section 7 we will show that our results hold $K(n)$-locally, i.e., that there are no non-trivial connected $K(n)$-local Galois extensions with finite Galois group at odd primes and with finite Galois group with cyclic quotient for the even prime.

So far, not many examples of separably closed commutative $S$-algebras are known. In [R, Theorem 10.3.3], Rognes proves that the (unlocalised) sphere spectrum is separably closed. His proof uses the fact that the ring of integers is separably closed, see [R, Proposition 10.3.2]. We show that $E_{n}^{\mathrm{nr}}$ is a separable closure of the sphere in the $K(n)$-local category for all $n$ and all odd primes $p$. Hovey and Strickland showed that the $K(n)$-local category is irreducible, i.e., it has no non-trivial localising (or colocalising) subcategories [HS, Section 7].

In [BR:3] we used the convention that for a Galois extension of commutative $S$ algebras $A \longrightarrow B$ it is assumed that $B$ is faithful as an $A$-module in the sense of $[\mathbf{R}$, Definition 4.3.1]. For the investigation of possible Galois extensions of $E_{n}^{\text {nr }}$ we do not need this assumption because we can exploit the fact that $E_{n}^{\text {nr }}$ has a residue field that is a finite cell $E_{n}^{\text {nr }}$-module spectrum. We are grateful to John Rognes who suggested that line of argument. Therefore a $G$-Galois extension of commutative $S$-algebras 
$A \longrightarrow B$ is understood to consist of the following data (compare [R, Definition 4.1.3] and [BR:3, Definition 1.4.4]).

Let $A$ be a commutative $S$-algebra and let $B$ be a commutative cofibrant $A$-algebra. Let $G$ be a finite (discrete) group and suppose that there is an action of $G$ on $B$ by commutative $A$-algebra morphisms. Then $B / A$ is a $G$-Galois extension if it satisfies the following two conditions:

- The natural map $i: A \longrightarrow B^{h G}=F\left(E G_{+}, B\right)^{G}$ is a weak equivalence of $A$ algebras.

- The canonical map of $B$-algebras

$$
h: B \wedge_{A} B \longrightarrow F\left(G_{+}, B\right)
$$

that is induced from the action of $G$ on the right hand factor of $B$ is an equivalence.

If $i$ and $h$ are $X$-equivalences for some spectrum $X$, then $B / A$ is called an $X$-local $G$-Galois extension.

\section{The spectrum $E_{n}^{\mathrm{nr}}$}

For ease of reference, we provide some details on the spectrum $E_{n}^{\mathrm{nr}}$, expanding on the discussion of [R, Section 5.4.1].

For each $k \geqslant 1$, using the methods of [SVW, BR:3], it follows that there is a Galois extension $W \mathbb{F}_{p^{n k}} E_{n} / E_{n}$ with Galois group $\operatorname{Gal}\left(\mathbb{F}_{p^{n k}} / \mathbb{F}_{p^{n}}\right) \cong C_{k}$ and

$$
\pi_{*}\left(W \mathbb{F}_{p^{n k}} E_{n}\right)=W \mathbb{F}_{p^{n k}} \otimes_{W \mathbb{F}_{p^{n}}} \pi_{*}\left(E_{n}\right) .
$$

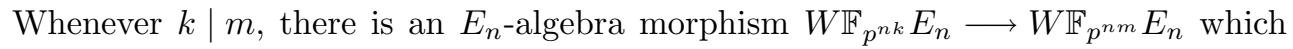
on homotopy groups induces the obvious homomorphism obtained from the natural inclusions $W \mathbb{F}_{p^{n k}} \longrightarrow W \mathbb{F}_{p^{n m}}$. Taking the colimit in the category of commutative $E_{n^{-}}$ algebras leads to a spectrum which is not $K(n)$-local, although each of the $W \mathbb{F}_{p^{n k}} E_{n}$ is $K(n)$-local since it is a finite wedge of copies of $E_{n}$. The homotopy ring

$$
\pi_{*}\left(\operatorname{colim}_{k} W \mathbb{F}_{p^{n k}} E_{n}\right) \cong \underset{k}{\operatorname{colim}} W \mathbb{F}_{p^{n k}}\left[\left[u_{1}, \ldots, u_{n-1}\right]\right]\left[u^{ \pm 1}\right]
$$

is Noetherian, regular and local. Let $K_{n}$ denote the $E_{n}$-module spectrum $E_{n} / \mathfrak{m}$ with $\mathfrak{m}$ denoting the maximal ideal $\left(p, u_{1}, \ldots, u_{n-1}\right)$ in $\pi_{0}\left(E_{n}\right)$. The finiteness of $K^{\prime}=\operatorname{colim}_{k} W \mathbb{F}_{p^{n k}} K_{n}$ over $E^{\prime}=\operatorname{colim}_{k} W \mathbb{F}_{p^{n k}} E_{n}$ ensures that we can apply [BL, Theorem 6.4] and obtain that

$$
L_{K^{\prime}}^{E^{\prime}} E^{\prime}=\underset{k}{\operatorname{holim}} E^{\prime} / \mathfrak{m}^{k}
$$

and

$$
\pi_{*} L_{K^{\prime}}^{E^{\prime}} E^{\prime}=\lim _{k}\left(\pi_{*} E^{\prime}\right) / \mathfrak{m}^{k}=W \overline{\mathbb{F}}_{p}\left[\left[u_{1}, \ldots, u_{n-1}\right]\right]\left[u^{ \pm 1}\right] .
$$

Finally, a $\Gamma$-cohomology obstruction theory argument similar to [RR, BR:1] applies to show that $L_{K^{\prime}}^{E^{\prime}} E^{\prime}=E_{n}^{\mathrm{nr}}$ does indeed have a unique commutative $E_{n}$-algebra structure.

An alternative way to construct $E_{n}^{\mathrm{nr}}$ is by considering the Honda formal group law over $\overline{\mathbb{F}}_{p}$ and its deformation theory with respect to the complete local ring 
$W \overline{\mathbb{F}}_{p}\left[\left[u_{1}, \ldots, u_{n-1}\right]\right]$. Then Goerss-Hopkins-Miller obstruction theory $[\mathbf{G H}, \S 7]$ shows that $E_{n}^{\mathrm{nr}}$ has a unique $E_{\infty}$-structure realizing $\left(E_{n}^{\mathrm{nr}}\right)_{*}\left(E_{n}^{\mathrm{nr}}\right)$ as a commutative $\pi_{*}\left(E_{n}^{\mathrm{nr}}\right)$ algebra and also that $E_{n}^{\mathrm{nr}}$ has an action of the group $\mathbb{S}_{n} \rtimes \widehat{\mathbb{Z}}$ via maps of $E_{\infty} \operatorname{ring}$ spectra. Rognes $[\mathbf{R}, 5.4 .6]$ shows that

$$
L_{K(n)} S \longrightarrow E_{n}^{\mathrm{nr}}
$$

is a $K(n)$-local profinite Galois extension.

\section{Some results on Galois theory}

We recall some facts about algebraic and topological Galois theory. We begin with some algebraic results about Galois extensions of graded commutative rings. In the following, $G$ will always be a finite group.

Let $R \longrightarrow S$ be a $G$-Galois extension of graded commutative rings. We include the following discussion along the lines of [CHR, Theorem 1.3] at the suggestion of the referee, because we do not know of any convenient source that states that in the context of graded Galois extensions $S$ is finitely generated projective over $R$. The impatient reader is invited to move on directly to Proposition 3.3.

The unramified condition gives an isomorphism of $S \otimes_{R} S$-modules

$$
h: S \otimes_{R} S \stackrel{\cong}{\longrightarrow} \prod_{G} S ; \quad x \otimes y \mapsto(x g(y))_{g \in G},
$$

where the bimodule structure on the right hand side is given by

$$
a\left(t_{g}\right) b=\left(a t_{g} g(b)\right)_{g \in G} \text {. }
$$

Then there is a map

$$
\sigma: S \longrightarrow S \otimes_{R} S ; \quad x \mapsto(x \otimes 1) h^{-1}\left(\delta_{1}\right),
$$

where for each $g \in G, \delta_{g}=\left(\delta_{g, h}\right)_{h \in G}$ is the element of $\prod_{G} S$ which has zeros everywhere except for a one in the entry corresponding to $g$. It is easy to see that $\sigma$ is a bimodule map and when composed with the product $\mu: S \otimes_{R} S \longrightarrow S$ we obtain $\mu \sigma=\mathrm{id}_{S}$. So $S$ is separable. In particular there is an idempotent

$$
e=h^{-1}\left(\delta_{1}\right) \in S \otimes_{R} S,
$$

and we can write

$$
e=\sum_{i} u_{i} \otimes v_{i}
$$

for some finite collection of elements $u_{i}, v_{i} \in S$. Notice that

$$
\delta_{1}=h(e)=\left(\sum_{i} u_{i} g\left(v_{i}\right)\right)_{g \in G},
$$

so for each $g \in G$ we have

$$
\sum_{i} u_{i} g\left(v_{i}\right)= \begin{cases}1 & \text { if } g=1 \\ 0 & \text { otherwise }\end{cases}
$$


Define the following $R$-linear maps:

$$
\alpha_{i}: S \longrightarrow R ; \quad x \mapsto \sum_{g \in G} g\left(v_{i}\right) g(x) .
$$

Calculating in $S$ we have

$$
\sum_{i} u_{i} \alpha_{i}(x)=\sum_{i} u_{i} \sum_{g \in G} g\left(v_{i}\right) g(x)=\sum_{g \in G}\left(\sum_{i} u_{i} g\left(v_{i}\right)\right) g(x)=x .
$$

Now we use a well known characterisation of finitely generated projective modules that applies as well in the graded case.

Lemma 3.1. Let $R$ be a graded commutative ring and let $M$ be a graded $R$-module. Then $M$ is a finitely generated projective module if and only if for some $n$ there are elements $b_{1}, \ldots, b_{n} \in M$ and $R$-linear maps $\alpha_{1}, \ldots, \alpha_{n}: M \longrightarrow R$ such that for every $x \in M$,

$$
x=\sum_{i} \alpha_{i}(x) b_{i}
$$

Thus we obtain the following result.

Lemma 3.2. $A$ G-Galois extension $R \longrightarrow S$ is a finitely generated projective $R$ module.

Proposition 3.3. Let $S / R$ be a $G$-Galois extension of graded commutative rings. Then for any graded commutative $R$-algebra $T, T \otimes_{R} S / T$ is also a $G$-Galois extension. In particular, if $I \triangleleft R$ is an ideal, $(S / S I) /(R / I)$ is a G-Galois extension.

Proof. A proof in the ungraded case can be found for instance in [CHR, Lemma 1.7] and with the help of Lemma 3.2 it carries over to the graded case.

In the following we need to understand base-change properties of topological Galois extensions.

Proposition 3.4. Let B/A be a G-Galois extension of commutative S-algebras. Suppose that $A \longrightarrow C$ is a map of commutative $S$-algebras and assume that $C$ is weakly equivalent to a retract of a finite cell $A$-module spectrum. Then $C \wedge_{A} B / C$ is also a G-Galois extension.

Proof. See [R, Lemma 7.1.3].

We need to understand base changes as above along $C$, where $C$ is a residue field in the sense of [BR:2] and which also happens to be an $A$-algebra. For instance, we could take $A=E_{n}^{\mathrm{nr}}$, the $n$-th Lubin-Tate spectrum, and $C=K_{n}^{\mathrm{nr}}$, the associated Morava $K$-theory with one of its strict multiplicative structures described in $[\mathbf{A}]$. However, in these cases $C$ is not a commutative $S$-algebra, but for our purposes it suffices that its coefficient ring is a graded commutative ring.

Corollary 3.5. Let $B$ be a cofibrant commutative A-algebra. Assume $\pi_{*}(B) / \pi_{*}(A)$ is a $G$-Galois extension and $C$ is an associative $A$-algebra whose coefficient ring $\pi_{*}(C)$ is a graded commutative $\pi_{*}(A)$-algebra. Then $\pi_{*}\left(C \wedge_{A} B\right) / \pi_{*}(C)$ is also a $G$-Galois extension. 
Proof. The assumption on the homotopy rings implies that $\pi_{*}(B)$ is a finitely generated projective $\pi_{*}(A)$-module. Therefore the relevant Künneth spectral sequence of $[\mathbf{E K M M}]$ collapses to give an isomorphism

$$
\pi_{*}\left(C \wedge_{A} B\right) \cong \pi_{*}(C) \otimes_{\pi_{*}(A)} \pi_{*}(B) .
$$

Now the result follows from Proposition 3.3.

Note that the realizability results of $[\mathbf{B R : 3}]$ imply that in the situation above the algebraic $G$-Galois extension $\pi_{*}(B) / \pi_{*}(A)$ can be realized by a $G$-Galois extension of commutative $S$-algebras $A \longrightarrow B^{\prime}$ with $B^{\prime} \simeq B$.

Proposition 3.6. Let $B / A$ be a $G$-Galois extension of commutative $S$-algebras. Let $C$ be an associative $A$-algebra that is a retract of a finite cell $A$-module spectrum and for which $\pi_{*}(C)$ is a graded field. Then $\pi_{*}\left(C \wedge_{A} B\right) / \pi_{*}(C)$ is an algebraic G-Galois extension.

Proof. The assumption that $C$ is a retract of a finite cell $A$-module spectrum guarantees that the homotopy fixed points $\left(C \wedge_{A} B\right)^{h G}$ are weakly equivalent to $C \wedge_{A}$ $B^{h G} \simeq C \wedge_{A} A \simeq C$ by $\left[\mathbf{R}\right.$, Lemma 6.2.6]. In particular, this shows that $C \wedge_{A} B$ is not contractible.

The unramified condition follows from the evident chain of isomorphisms

$$
\begin{aligned}
\pi_{*}\left(C \wedge_{A} B\right) \otimes_{\pi_{*}(C)} \pi_{*}\left(C \wedge_{A} B\right) \stackrel{\cong}{\cong} \pi_{*}\left(C \wedge_{A} B \wedge_{A} B\right) \\
\stackrel{\cong}{\cong} \pi_{*}\left(\prod_{G} C \wedge_{A} B\right) \stackrel{\cong}{\longrightarrow} \prod_{G} \pi_{*}\left(C \wedge_{A} B\right) .
\end{aligned}
$$

We know that $\pi_{*}\left(C \wedge_{A} B\right)$ is a finite dimensional $\pi_{*}(C)$-vector space. Let $k$ be the dimension of $\pi_{*}\left(C \wedge_{A} B\right)$ over $\pi_{*}(C)$. The $G$-equivariant isomorphism

$$
\pi_{*}\left(C \wedge_{A} B\right) \otimes_{\pi_{*}(C)} \pi_{*}\left(C \wedge_{A} B\right) \cong \prod_{G} \pi_{*}\left(C \wedge_{A} B\right)
$$

maps $\pi_{*}\left(C \wedge_{A} B\right) \otimes_{\pi_{*}(C)} \pi_{*}\left(C \wedge_{A} B\right)^{G}$ isomorphically onto a subspace of

$$
\left(\prod_{G} \pi_{*}\left(C \wedge_{A} B\right)\right)^{G} \cong \pi_{*}\left(C \wedge_{A} B\right)
$$

Note that this shows that $k^{2}=k|G|$ and thus $k$ is the cardinality of the group. Now we know that

$$
\operatorname{dim}_{\pi_{*}(C)} \pi_{*}\left(C \wedge_{A} B\right) \otimes_{\pi_{*}(C)} \pi_{*}\left(C \wedge_{A} B\right)^{G}=k \operatorname{dim}_{\pi_{*}(C)} \pi_{*}\left(C \wedge_{A} B\right)^{G} \leqslant k
$$

and therefore $\pi_{*}\left(C \wedge_{A} B\right)^{G}$ is 1-dimensional over $\pi_{*}(C)$.

In our work we will need a basic lemma on idempotents in Galois extensions. For background on idempotents on commutative $S$-algebras, see $[\mathbf{R}, 10.2]$. We just recall some of the main results.

Let $R$ be a commutative $S$-algebra; then we say that a commutative $R$-algebra $A$ splits if there is a weak equivalence of commutative $R$-algebras $A \simeq A_{1} \times A_{2}$ for some commutative $R$-algebras $A_{1}, A_{2}$ which satisfy $A_{1} \nsucceq * \nsucc A_{2}$, i.e., they are homotopically non-trivial as commutative $R$-algebras. If $A$ admits no such splitting it is said to be connected, otherwise it is non-connected. 
Let $\mathcal{E}(A)$ denote the mapping space of non-unital commutative $A$-algebra endomorphisms of $A$. Rognes shows in [R, Lemma 10.2.3] that $A$ is connected if and only if the map of spaces $\{0,1\} \longrightarrow \mathcal{E}(A)$ that takes 0 to the constant map and 1 to the identity map is a weak equivalence. Furthermore he proves in [R, Proposition 10.2.2] that $\pi_{0} \mathcal{E}(A)$ corresponds to the idempotents of the ring $\pi_{0}(A)$, thus $A$ is connected if and only if $\pi_{0}(A)$ is connected in the sense of algebra.

Lemma 3.7. Let $\iota: A \longrightarrow B$ be a $G$-Galois extension of commutative $S$-algebras. If $A$ splits as $A \simeq A_{1} \times A_{2}$, then as a commutative $A$-algebra, $B$ splits as $B \simeq B_{1} \times B_{2}$, such that there are compatible maps of commutative $S$-algebras $A_{1} \longrightarrow B_{1}$ and $A_{2} \longrightarrow$ $B_{2}$ which are $G$-Galois extensions.

We are grateful to the referee who replaced our earlier clumsier proof by the following straightforward line of argument.

Proof. Let $B_{i}=A_{i} \wedge_{A} B$ for $i=1,2$. Since $A_{1}$ and $A_{2}$ are retracts of a finite cell $A$-module, namely $A$ itself, $A_{i} \longrightarrow B_{i}$ is a $G$-Galois extension for $i=1,2$ by Proposition 3.4. The equivalence of commutative $S$-algebras $A \rightarrow A_{1} \times A_{2}$ induces the equivalence

$$
B \simeq A \wedge_{A} B \rightarrow\left(A_{1} \times A_{2}\right) \wedge_{A} B
$$

and the latter is equivalent to

$$
\left(A_{1} \vee A_{2}\right) \wedge_{A} B \simeq A_{1} \wedge_{A} B \vee A_{2} \wedge_{A} B=B_{1} \vee B_{2} \simeq B_{1} \times B_{2} .
$$

\section{Calculations with residue fields}

We recall from $[\mathbf{B R : 2}, \S 3]$ the notion of a residue field for a commutative $S$-algebra $R$. Let $\mathfrak{m}$ be a maximal ideal in $\pi_{*}(R)$. If there is an $R$-module spectrum $W$ for which the $\pi_{*}(R)$-module $\pi_{*}(W)$ is isomorphic to $\pi_{*}(R) / \mathfrak{m}$, then we call $W$ a residue field of $R$ with respect to $\mathfrak{m}$. Note that in general no multiplicative structure on $W$ is assumed.

In the case of $E_{n}^{\mathrm{nr}}$, there is a version of the Morava $K$-theory spectrum $K_{n}^{\mathrm{nr}}$ with coefficient ring

$$
\pi_{*}\left(K_{n}^{\mathrm{nr}}\right)=\pi_{*}\left(E_{n}^{\mathrm{nr}}\right) / \mathfrak{m}=\overline{\mathbb{F}}_{p}\left[u, u^{-1}\right] .
$$

Thus $\pi_{*}\left(K_{n}^{\mathrm{nr}}\right)$ is the residue field of the local ring $\pi_{*}\left(E_{n}^{\mathrm{nr}}\right)$ and so $K_{n}^{\mathrm{nr}}$ is a residue field for $E_{n}^{\mathrm{nr}}$. By work of $[\mathbf{A}], K_{n}^{\mathrm{nr}}$ admits the structure of an associative $E_{n}^{\mathrm{nr}}$-algebra.

Notation. To simplify notation, we set $E=E_{n}^{\mathrm{nr}}$ and $K=K_{n}^{\mathrm{nr}}$ from now on.

For two $E$-modules $M, N$, at least one of which is cofibrant, there is a Künneth spectral sequence

$$
\mathrm{E}_{s, t}^{2}=\operatorname{Tor}_{s, t}^{\pi_{*}(K)}\left(\pi_{*}\left(K \wedge_{E} M\right), \pi_{*}\left(K \wedge_{E} N\right)\right) \Longrightarrow \pi_{*}\left(K \wedge_{E} M \wedge_{E} N\right)
$$

which collapses to give a Künneth isomorphism of $\pi_{*}(K)$-modules

$$
\pi_{*}\left(K \wedge_{E} M \wedge_{E} N\right) \cong \pi_{*}\left(K \wedge_{E} M\right) \otimes_{\pi_{*}(K)} \pi_{*}\left(K \wedge_{E} N\right)
$$

Since these homotopy groups are 2 -periodic, we can view them as $\mathbb{Z} / 2$-graded modules. For a $K$-module $V, \pi_{*}(V)$ is equivalent to a $\mathbb{Z} / 2$-graded $\overline{\mathbb{F}}_{p}$-vector space 
and then we can consider the dimensions of the even and the odd parts separately. For an $E$-module spectrum $M$ we set

$$
d_{0}=\operatorname{dim}_{\overline{\mathbb{F}}_{p}} \pi_{0}\left(K \wedge_{E} M\right), \quad d_{1}=\operatorname{dim}_{\overline{\mathbb{F}}_{p}} \pi_{1}\left(K \wedge_{E} M\right) .
$$

Lemma 4.1. Suppose that an E-module spectrum $M$ satisfies

$$
M \wedge_{E} M \simeq \prod_{X} M
$$

for some finite set $X$ of cardinality $|X|=m$. If $\pi_{*}\left(K \wedge_{E} M\right)$ is a non-trivial finite dimensional $\pi_{*}(K)$-module, then the dimensions $d_{0}$ and $d_{1}$ satisfy one of the following conditions:

- $d_{1}=0$ and $d_{0}=m$.

- $d_{1} \neq 0, m$ is even and $d_{0}=m / 2=d_{1}$.

In particular, if $m$ is odd, then we must have the first condition.

Proof. Using the Künneth formula based on $K$, we have

$$
\operatorname{dim}_{\overline{\mathbb{F}}_{p}} \pi_{0}\left(K \wedge_{E} M \wedge_{E} M\right)=d_{0}^{2}+d_{1}^{2}, \quad \operatorname{dim}_{\overline{\mathbb{F}}_{p}} \pi_{1}\left(K \wedge_{E} M \wedge_{E} M\right)=2 d_{0} d_{1} .
$$

On the other hand, by the assumed splitting of $M \wedge_{E} M$ we obtain the equations

$$
d_{0}^{2}+d_{1}^{2}=m d_{0}, \quad 2 d_{0} d_{1}=m d_{1} .
$$

Using these we establish the result.

\section{Separable closure property at odd primes}

For an odd prime $p$ we can prove a general result.

Theorem 5.1. Let $G$ be an arbitrary finite group and $p$ an odd prime. Then for every $G$-Galois extension $B$ of $E$ there is a weak equivalence of commutative E-algebras

$$
B \simeq \prod_{G} E
$$

Proof. We know from Proposition 3.6 that the Galois extension $E \longrightarrow B$ gives rise to a $G$-Galois extension $\pi_{*}(K) \longrightarrow \pi_{*}\left(K \wedge_{E} B\right)$ of graded rings, in particular, $\pi_{*}\left(K \wedge_{E}\right.$ $B)$ is a graded separable $\pi_{*}(K)$-algebra. DeMeyer and Ingraham showed in [DeMI, Proposition II.2.3] that for a separable (ungraded) algebra $A$ over a commutative ring $R$ any $R$-projective $A$-module $M$ is also $A$-projective. Their proof translates to the graded setting without any changes, thus any $\pi_{*}(K)$-projective $\pi_{*}\left(K \wedge_{E} B\right)$ module is also $\pi_{*}\left(K \wedge_{E} B\right)$-projective. Assume that $z$ is a non-trivial element in $\pi_{1}\left(K \wedge_{E} B\right)$. Then the cyclic $\pi_{*}\left(K \wedge_{E} B\right)$-submodule $\pi_{*}\left(K \wedge_{E} B\right) z \subseteq \pi_{*}\left(K \wedge_{E} B\right)$ is projective and so the surjection $\varphi: \pi_{*}\left(K \wedge_{E} B\right) \longrightarrow \pi_{*}\left(K \wedge_{E} B\right) z$ given by $\varphi(a)=$ $a z$ is split by a $\pi_{*}\left(K \wedge_{E} B\right)$-homomorphism $\rho: \pi_{*}\left(K \wedge_{E} B\right) z \longrightarrow \pi_{*}\left(K \wedge_{E} B\right)$ under which $\rho(z)=1+w$ for some $w$ satisfying $w z=0$. Using $\pi_{*}\left(K \wedge_{E} B\right)$-linearity we obtain

$$
\rho\left(z^{2}\right)=z(1+w)=z+z w=z \neq 0 .
$$

But we are working in odd characteristic, hence $z^{2}=0$. Therefore the odd part of $\pi_{*}\left(K \wedge_{E} B\right)$ has to vanish. 
Let $K^{\prime}$ be the $E$-module spectrum $E /\left(p, u_{1}, \ldots, u_{n-2}\right)$. We consider the long exact sequence corresponding to the cofibre sequence

$$
K^{\prime} \wedge_{E} B \stackrel{u_{n-1}}{\longrightarrow} K^{\prime} \wedge_{E} B \longrightarrow K \wedge_{E} B
$$

As we know from above, $\pi_{*}\left(K \wedge_{E} B\right)$ is concentrated in even degrees. Thus we obtain the exactness of

$$
\begin{array}{r}
0 \rightarrow \pi_{2 q}\left(K^{\prime} \wedge_{E} B\right) \stackrel{u_{n-1}}{\longrightarrow} \pi_{2 q}\left(K^{\prime} \wedge_{E} B\right) \stackrel{j}{\rightarrow} \pi_{2 q}\left(K \wedge_{E} B\right) \stackrel{\delta}{\rightarrow} \pi_{2 q-1}\left(K^{\prime} \wedge_{E} B\right) \\
\stackrel{u_{n-1}}{\longrightarrow} \pi_{2 q-1}\left(K^{\prime} \wedge_{E} B\right) \rightarrow 0 .
\end{array}
$$

The associated Bockstein spectral sequence (see [We, 5.9.9]) has $B_{*}^{0}=\pi_{*}\left(K \wedge_{E}\right.$ $B)=0$ in odd degrees and all differentials have degree -1 and are therefore trivial. Denote $\pi_{*}\left(K^{\prime} \wedge_{E} B\right)$ by $A_{*}^{0}$. Then we obtain a short exact sequence

$$
0 \rightarrow A_{i}^{0} /\left(u_{n-1} A_{i}^{0}+{ }_{u_{n-1}^{r}} A_{i}^{0}\right) \stackrel{\bar{j}^{r}}{\longrightarrow} B_{i}^{r} \stackrel{\delta^{r}}{\longrightarrow} u_{n-1} A_{i-1}^{0} \cap u_{n-1}^{r} A_{i-1}^{0} \rightarrow 0
$$

for every $r \geqslant 0$. As we have that the evenly graded part of $A_{*}^{0}$ is $u_{n-1}$-torsion free and that

$$
B_{*}^{0}=B_{*}^{1}=\cdots=B_{*}^{\infty},
$$

this yields that for an even degree $i=2 q$ we have

$$
u_{n-1} A_{2 q-1}^{0}={ }_{u_{n-1}} A_{2 q-1}^{0} \cap u_{n-1}^{r} A_{2 q-1}^{0}
$$

for all $r \geqslant 0$. As $\pi_{*}\left(K^{\prime} \wedge_{E} B\right)$ is a finitely generated $\pi_{*}(E)$-module, we can deduce that $\pi_{2 q-1}\left(K^{\prime} \wedge_{E} B\right)$ is $u_{n-1}$-torsion free. But then the multiplication with $u_{n-1}$ has to be an isomorphism on $\pi_{2 q-1}\left(K^{\prime} \wedge_{E} B\right)$ so we see that $\pi_{2 q-1}\left(K^{\prime} \wedge_{E} B\right)$ is actually trivial for all $q$ and

$$
\pi_{0}\left(K \wedge_{E} B\right) \cong \pi_{0}\left(K^{\prime} \wedge_{E} B\right) /\left(u_{n-1}\right) .
$$

Considering the spectra $E /\left(p, u_{1}, \ldots, u_{j}\right)$ for $j=n-3, \ldots, 0$ (with $p=u_{0}$ ) in a similar fashion we obtain

$$
\pi_{0}\left(K \wedge_{E} B\right) \cong \pi_{0}(B) /\left(p, u_{1}, \ldots, u_{n-1}\right) \cong \pi_{0}(K) \otimes_{\pi_{0}(E)} \pi_{0}(B) .
$$

The quotient $\pi_{0}(B) / \mathfrak{m}$ is a separable extension of the separably closed field $\overline{\mathbb{F}}_{p}$, so by [DeMI, Corollary II.2.4], this extension has to split as

$$
\pi_{0}\left(K \wedge_{E} B\right) \cong \prod_{G} \overline{\mathbb{F}}_{p}
$$

As $B$ is a finite cell $E$-module we know that $\pi_{0}(B)$ is a finitely generated module over the Noetherian ring $\pi_{0}(E)$ and the calculation above shows that

$$
\pi_{0}\left(K \wedge_{E} B\right) \cong \pi_{0}(B) / \mathfrak{m}
$$

By the lifting of idempotents result of $[\mathbf{E}$, Corollary 7.5] for instance, the orthogonal idempotents that give rise to this splitting lift to the $\pi_{0}(E)$-algebra $\pi_{0}(B)$ and we obtain the desired splitting of $B$ into $G$ copies of $E$. 


\section{Galois groups with cyclic quotients}

We will consider Galois extensions of $E$ with Galois groups having finite cyclic quotients. We note that the result concerning these extensions is valid for all primes.

Theorem 6.1. Let $B / E$ be a $G$-Galois extension where $G$ is a finite group with a cyclic quotient of prime order. Then $B$ is non-connected.

Corollary 6.2. Every $G$-Galois extension $B$ of $E$ with finite solvable Galois group $G$ is non-connected. In this sense, the commutative $E_{n}$-algebra $E$ is a maximal connected solvable Galois extension of $E_{n}$.

Of course for odd primes this result is covered by Theorem 5.1. We include a complete proof for all primes, because a reduction to the case $p=2$ does not yield a much shorter proof and we feel that the full proof offers some insight.

Proof. By assumption, there is a normal subgroup $N \triangleleft G$ for which one of the following holds:

(A) $G / N \cong C_{\ell}$ with $\ell$ a prime different from $p$,

(B) $G / N \cong C_{p}$.

Case (A): We consider the extension $\pi_{*}(K) \longrightarrow \pi_{*}\left(K \wedge_{E} B^{h N}\right)$ in which $\pi_{*}\left(K \wedge_{E}\right.$ $\left.B^{h N}\right)$ is a finite dimensional algebra over the graded field $\pi_{*}(K)$ and is an algebraic $C_{\ell^{-}}$Galois extension by Proposition 3.6.

The argument of [BR:2, Example 42] shows that the homotopy groups $\pi_{*}\left(K \wedge_{E}\right.$ $\left.B^{h N}\right)$ have to be concentrated in even degrees: as $\pi_{*}\left(K \wedge_{E} B^{h N}\right)$ is a $C_{\ell^{-G a l o i s}}$ extension of $\pi_{*} K$, each homotopy group $\pi_{2 n+1}\left(K \wedge_{E} B^{h N}\right)$ is a $C_{\ell^{-}}$-representation and it has a decomposition into character eigenspaces because $\ell \neq p$. If there were odd-degree elements and if $p$ is an odd prime then the map $x_{1} \otimes x_{2} \mapsto\left(x_{1} g\left(x_{2}\right)\right)_{g \in G}$ would have a non-trivial kernel, thus contradicting the unramified condition. For $p=2$, every irreducible character has odd order, and thus an odd degree element of the corresponding summand is nilpotent, because some odd power lies in the invariant part which is trivial in odd degrees. If $x$ is such an element with $x^{j}=0$, then $x^{j-1} \otimes x$ would be in the kernel of the above mentioned map, because $x^{j-1} g(x)=\lambda_{g} x^{j}=0$, where $g(x)=\lambda_{g} x$.

Thus we can focus on the extension $\pi_{0}\left(K \wedge_{E} B^{h N}\right) / \pi_{0}(K)$. Now $\pi_{0}(K)=\overline{\mathbb{F}}_{p}$ is a separably closed field, so this extension has to split completely, i.e.,

$$
\pi_{0}\left(K \wedge_{E} B^{h N}\right) \cong \prod_{C_{\ell}} \overline{\mathbb{F}}_{p}
$$

We know that $\pi_{0}\left(B^{h N}\right)$ is a finitely generated $\pi_{0}(E)$-module, and $\pi_{0}(E)$ is complete with respect to the maximal ideal $\mathfrak{m} \triangleleft \pi_{0}(E)$. A Bockstein spectral sequence argument similar to the proof of Theorem 5.1 shows that $\pi_{0}\left(K \wedge_{E} B^{h N}\right) \cong \pi_{0}\left(B^{h N}\right) / \mathfrak{m}$. Therefore by the usual lifting of idempotents result of $[\mathbf{E}$, Corollary 7.5], the $\ell$ orthogonal idempotents that give rise to this splitting lift to the $\pi_{0}(E)$-algebra $\pi_{0}\left(B^{h N}\right)$. Thus 
we obtain a splitting of $\pi_{0}(E)$-algebras

$$
\pi_{0}\left(B^{h N}\right) \cong \prod_{C_{\ell}} \pi_{0}(E)
$$

and accordingly

$$
B^{h N} \simeq \prod_{C_{\ell}} E
$$

so we see that $B^{h N}$ splits completely. Now by Lemma 3.7, in Case (A) we obtain a non-trivial splitting of $B$.

Case (B): When $G / N \cong C_{p}$, we consider two cases.

First let us assume that

$$
\operatorname{dim}_{\overline{\mathbb{F}}_{p}} \pi_{0}\left(K \wedge_{E} B^{h N}\right)=p, \quad \operatorname{dim}_{\overline{\mathbb{F}}_{p}} \pi_{1}\left(K \wedge_{E} B^{h N}\right)=0 .
$$

By Proposition 3.6, the pair $\pi_{0}\left(K \wedge_{E} B^{h N}\right) / \pi_{0}(K)$ forms an algebraic $C_{p}$-Galois extension. As in Case (A), we can now deduce that this extension splits, so

$$
\pi_{0}\left(K \wedge_{E} B^{h N}\right) \cong \prod_{C_{p}} \pi_{0}(K) .
$$

From this isomorphism we obtain $p$ orthogonal idempotents in $\pi_{0}\left(K \wedge_{E} B^{h N}\right)$, each realised by a map

$$
K \longrightarrow K \wedge_{E} B^{h N}
$$

Again we can lift the idempotents that cause this splitting because of the completeness of $\pi_{0}(E)$ and therefore we can realise the corresponding splitting as

$$
B^{h N} \simeq \prod_{G} E
$$

For $p=2$, according to Lemma 4.1 we have to exclude the possibility that

$$
\operatorname{dim}_{\overline{\mathbb{F}}_{p}} \pi_{0}\left(K \wedge_{E} B^{h N}\right)=1, \quad \operatorname{dim}_{\overline{\mathbb{F}}_{p}} \pi_{1}\left(K \wedge_{E} B^{h N}\right)=1 .
$$

As the $C_{2}$-action preserves degree, we know that

$$
\operatorname{dim}_{\overline{\mathbb{F}}_{p}} \pi_{0}\left(K \wedge_{E} B^{h N}\right)^{C_{2}}=1, \quad \operatorname{dim}_{\overline{\mathbb{F}}_{p}} \pi_{1}\left(K \wedge_{E} B^{h N}\right)^{C_{2}}=0
$$

because $\pi_{*}(K)$ is concentrated in even degrees. Thus the $C_{2}$-action on $\pi_{0}\left(K \wedge_{E} B^{h N}\right)$ has fixed points, whereas the action on $\pi_{1}\left(K \wedge_{E} B^{h N}\right)$ must have no non-trivial fixed points.

To finish, we adapt the argument in the proof of [Sh, Proposition 17] to show that $\pi_{1}\left(K \wedge_{E} B^{h N}\right)$ must be trivial: If a finite $p$-group $P$ acts on an arbitrary abelian $p$ torsion group $M$, then for any non-zero element $x \in M$, the subgroup of $M$ generated by the $P$-orbit of $x$ is a finite $p$-group which is also a $P$-submodule and so by $[\mathbf{S h}$, Proposition 17] it has non-trivial fixed points.

Thus in each of Cases $(\mathrm{A})$ and $(\mathrm{B}), B^{h N}$ is not connected and therefore by using Lemma 3.7, we see that $B$ as an $N$-Galois extension of $B^{h N}$ is not connected either. 


\section{The $K(n)$-local case}

Again we let $(E, K)$ be the pair $\left(E_{n}^{\mathrm{nr}}, K_{n}^{\mathrm{nr}}\right)$, but note that the discussion in this section carries over to the pairs $\left(E_{n}, K_{n}\right)$ and $(\widehat{E(n)}, K(n))$ as well. Let $\mathcal{D}_{E}$ denote the stable homotopy category of $E$-module spectra and let $\mathcal{D}_{E, K}$ be the full subcategory generated by $K$-local $E$-modules. We denote the localization functor from $\mathcal{D}_{E}$ to $\mathcal{D}_{E, K}$ by $L=L_{E, K}$.

Remark 7.1. We note that by [Ho, Proposition 2.2], for $X \in \mathcal{D}_{E}$ (which gives rise to an element $X \in \mathcal{D}_{S}$ by restriction of scalars)

$$
X \in \mathcal{D}_{E, K} \quad \Longleftrightarrow \quad X \in \mathcal{D}_{S, K} .
$$

This implies that for an $E$-module spectrum $X$ the two conditions $K \wedge X \simeq *$ and $K \wedge_{E} X \simeq *$ are equivalent.

The aim of the following is to provide a reference for the fact that dualizable objects in $\mathcal{D}_{E, K}$ are retracts of finite cell $E$-modules. The argument we present here is due to Mark Hovey and we are grateful to him for allowing us to include it here. We recall from [HS, Definition 1.5] the definitions of dualizable and $F$-small.

We note that $K \wedge_{E} X$ is in $\mathcal{D}_{E, K}$, because it is a $K$-module spectrum and therefore it is $K$-local. We also know that $K$ is small in $\mathcal{D}_{E, K}$ since it is a finite cell $E$-module. This can be seen by expressing it as $K=E / \mathfrak{m}$ with $\mathfrak{m}=\left(p, u_{1}, \ldots, u_{n-1}\right)$, where the generating sequence of $\mathfrak{m}$ is regular. More generally there are finite cell $E$-module spectra $E / \mathfrak{m}^{s}(s \geqslant 1)$ which fit together to form the $\mathfrak{m}$-adic tower

$$
K=E / \mathfrak{m} \longleftarrow E / \mathfrak{m}^{2} \longleftarrow E / \mathfrak{m}^{3} \longleftarrow \cdots
$$

constructed in full generality in $[\mathbf{B L}]$. By $[\mathbf{W} \ddot{\mathbf{u}}$, Theorem 1.1], this is can be constructed as a tower of associative $E$-algebras. The fibre of the map $E / \mathfrak{m}^{s+1} \longrightarrow E / \mathfrak{m}^{s}$ is a finite wedge of suspensions of $K$, and this can be used to show that the Bousfield classes of $E / \mathfrak{m}^{s}$ and $K$ coincide.

We also remark that if $X$ is a retract of a finite cell $E$-module, then $\pi_{*}(X)$ is a finitely generated $\pi_{*}(E)$-module since $\pi_{*}(E)$ is Noetherian; the converse also holds since $\pi_{*}(E)$ is a regular local ring.

Lemma 7.2. If $X$ is dualizable in $\mathcal{D}_{E, K}$, then for an arbitrary indexing set $A$ and $Y_{\alpha} \in \mathcal{D}_{E, K}$, the natural map

$$
L \bigvee_{\alpha \in A} F_{E}\left(X, Y_{\alpha}\right) \longrightarrow F_{E}\left(X, L \bigvee_{\alpha \in A} Y_{\alpha}\right)
$$

is an isomorphism, i.e., $X$ is $F$-small in $\mathcal{D}_{E, K}$ in the sense of [HS, Definition 1.5].

Proof. As $X$ is dualizable in $\mathcal{D}_{E, K}$, we obtain

$$
\begin{aligned}
F_{E}\left(X, L \bigvee_{\alpha \in A} Y_{\alpha}\right) & \cong L\left(F_{E}(X, E) \wedge_{E} L \bigvee_{\alpha \in A} Y_{\alpha}\right) \\
& \cong L \bigvee_{\alpha \in A}\left(F_{E}(X, E) \wedge_{E} Y_{\alpha}\right) \\
& \cong L \bigvee_{\alpha \in A} F_{E}\left(X, Y_{\alpha}\right)
\end{aligned}
$$


Lemma 7.3. If $X$ is $F$-small in $\mathcal{D}_{E, K}$, then $K \wedge_{E} X$ is also $F$-small in $\mathcal{D}_{E, K}$.

Proof. The standard adjunction yields

$$
\left[K \wedge_{E} X, L \bigvee_{\alpha \in A} Y_{\alpha}\right]=\left[K, F_{E}\left(X, L \bigvee_{\alpha \in A} Y_{\alpha}\right)\right]
$$

As $X$ is $F$-small this coincides with $\left[K, L \bigvee_{\alpha \in A} F_{E}\left(X, Y_{\alpha}\right)\right]$ and the smallness of $K$ turns this into

$$
\bigoplus_{\alpha \in A}\left[K, F_{E}\left(X, Y_{\alpha}\right)\right] \cong \bigoplus_{\alpha \in A}\left[K \wedge_{E} X, Y_{\alpha}\right]
$$

Lemma 7.4. If $K \wedge_{E} X$ is small in $\mathcal{D}_{E, K}$, then $\pi_{*}\left(K \wedge_{E} X\right)$ is a finite-dimensional $\pi_{*}(K)$-vector space.

Proof. As $K$ is a field spectrum $K \wedge_{E} X$ splits as

$$
K \wedge_{E} X \cong \bigvee_{i} \Sigma^{\nu_{i}} K
$$

Thus, if $K \wedge_{E} X$ is small, the isomorphism

$$
K \wedge_{E} X \longrightarrow \bigvee_{i} \Sigma^{\nu_{i}} K=L\left(\bigvee_{i} \Sigma^{\nu_{i}} K\right)
$$

factors through a finite subwedge and hence the wedge must be finite.

Lemma 7.5. If $X \in \mathcal{D}_{E}$, then $L X$ is isomorphic to the homotopy limit

$$
\operatorname{holim}\left(E / \mathfrak{m}^{s} \wedge_{E} X\right)
$$

taken in the category of E-module spectra.

Proof. Each term $E / \mathfrak{m}^{s} \wedge_{E} X$ is an $E / \mathfrak{m}^{s}$-module spectrum and therefore it is $E / \mathfrak{m}^{s}$ local which is equivalent to being $K$-local. As we know that the Bousfield classes of $E / \mathfrak{m}^{s}$ and $K=E / \mathfrak{m}$ coincide for all $s \geqslant 1$, the homotopy limit $\underset{s}{\operatorname{holim}}\left(E / \mathfrak{m}^{s} \wedge_{E} X\right)$ is $K$-local.

We know that $K \wedge_{E} X$ is already $K$-local and the image of the reduction homomorphism

$$
\pi_{*}\left(K \wedge_{E} E / \mathfrak{m}^{s+1}\right) \longrightarrow \pi_{*}\left(K \wedge_{E} E / \mathfrak{m}^{s}\right)
$$

is $\pi_{*}(K)$ by [BL, Corollary 5.11], hence using the commutative diagram coming from the Künneth isomorphism

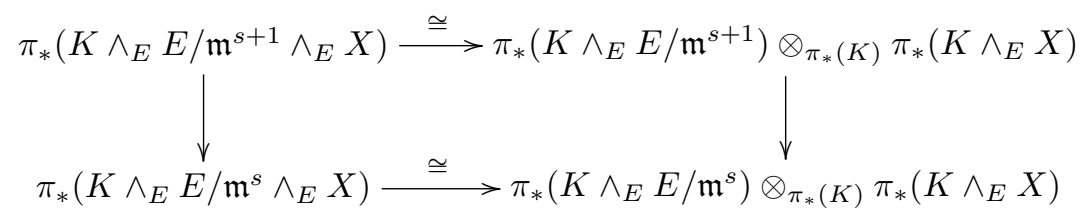

we find that

$$
\pi_{*}\left(\operatorname{holim}\left(E / \mathfrak{m}^{n} \wedge_{E} K \wedge_{E} X\right)\right) \cong \pi_{*}\left(K \wedge_{E} X\right)
$$


Therefore the map $X \longrightarrow \underset{s}{\operatorname{holim}}\left(E / \mathfrak{m}^{s} \wedge_{E} X\right)$ is a $K$-equivalence because

$$
\begin{aligned}
K \wedge_{E} \operatorname{holim}_{s}\left(E / \mathfrak{m}^{s} \wedge_{E} X\right) & \cong \operatorname{holim}_{s}\left(K \wedge_{E} E / \mathfrak{m}^{s} \wedge_{E} X\right) \\
& \cong K \wedge_{E} X
\end{aligned}
$$

Lemma 7.6. If $X$ is in $\mathcal{D}_{E, K}$, then its homotopy $\pi_{*}(X)$ is an L-complete $\pi_{*}(E)$ module in the sense of [HS, Definition A.5].

Proof. Each term $\pi_{*}\left(E / \mathfrak{m}^{s} \wedge_{E} X\right)$ is bounded $\mathfrak{m}$-torsion and hence it is $L$-complete. But the class of $L$-complete modules is closed under limits, $\lim ^{1}$-terms and extensions [HS, Theorem A.6], and therefore the Milnor sequence yields the result.

Lemma 7.7. If $X \in \mathcal{D}_{E, K}$ and $\pi_{*}\left(K \wedge_{E} X\right)$ is finite-dimensional over $\pi_{*} K$, then $\pi_{*}(X)$ is finitely generated over $\pi_{*}(E)$. In particular, $X$ is a retract of a finite cell E-module.

Proof. We prove that $\pi_{*}\left(E /\left(p, u_{1}, \ldots, u_{i}\right) \wedge_{E} X\right)$ is finitely generated over $\pi_{*}(E)$ by downward induction on $i$. The case $i=n-1$ is guaranteed by the assumption because $\pi_{*}(K)$ is finitely generated over $\pi_{*}(E)$.

We set

$$
X /\left(p, u_{1}, \ldots, u_{i}\right)=E /\left(p, u_{1}, \ldots, u_{i}\right) \wedge_{E} X .
$$

Then there is a long exact sequence of homotopy groups induced by the cofibre sequence

$$
X /\left(p, u_{1}, \ldots, u_{i-1}\right) \stackrel{u_{i}}{\longrightarrow} X /\left(p, u_{1}, \ldots, u_{i-1}\right) \longrightarrow X /\left(p, u_{1}, \ldots, u_{i}\right) .
$$

Denoting the annihilator of $u_{i}$ in $\pi_{*}\left(X /\left(p, u_{1}, \ldots, u_{i-1}\right)\right)$ by

$$
\operatorname{ann}\left(u_{i}, \pi_{*}\left(X /\left(p, u_{1}, \ldots, u_{i-1}\right)\right),\right.
$$

the long exact sequence yields the short exact sequence

$$
\begin{aligned}
0 \rightarrow \pi_{*}\left(X /\left(p, u_{1}, \ldots, u_{i-1}\right)\right) / u_{i} & \longrightarrow \pi_{*}\left(X /\left(p, u_{1}, \ldots, u_{i}\right)\right) \\
& \longrightarrow \operatorname{ann}\left(u_{i}, \pi_{*}\left(X /\left(p, u_{1}, \ldots, u_{i-1}\right)\right) \rightarrow 0 .\right.
\end{aligned}
$$

Since it injects into $\pi_{*}\left(X /\left(p, u_{1}, \ldots, u_{i}\right)\right), \pi_{*}\left(X /\left(p, u_{1}, \ldots, u_{i-1}\right)\right) / u_{i}$ is finitely generated. Hence there is a free $\pi_{*}(E)$-module $F_{*}$ together with a map

$$
f: F_{*} \longrightarrow \pi_{*}\left(X /\left(p, u_{1}, \ldots, u_{i-1}\right)\right)
$$

such that the induced map

$$
F_{*} \longrightarrow \pi_{*}\left(X /\left(p, u_{1}, \ldots, u_{i-1}\right)\right) / u_{i}
$$


is surjective. Let $N$ be the cokernel of $f$. The diagram of exact sequences

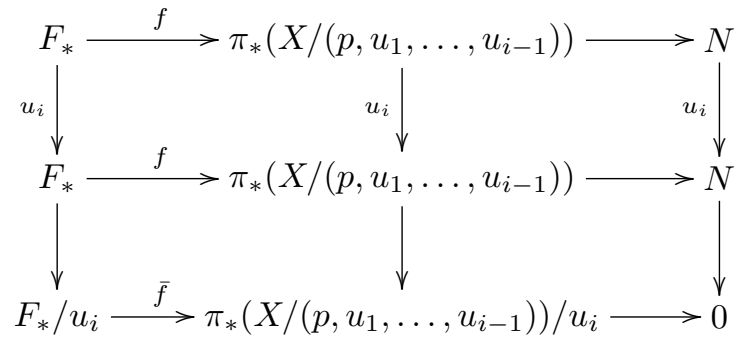

tells us that $u_{i} N=N$ and $L$-completeness of $N$ implies that $N=0$. Hence the map $F \longrightarrow \pi_{*}\left(X /\left(p, u_{1}, \ldots, u_{i-1}\right)\right)$ is surjective, and so $\pi_{*}\left(X /\left(p, u_{1}, \ldots, u_{i-1}\right)\right)$ is finitely generated over $\pi_{*}(E)$ for all $0 \leqslant i \leqslant n-1$.

As $\pi_{*}(E)$ is a regular local ring, this implies that $X$ is a retract of a finite cell E-module.

Lemma 7.8. Let $G$ be a finite group. If $E \longrightarrow B$ is a $K(n)$-local $G$-Galois extension, then

$$
\pi_{*}(K) \longrightarrow \pi_{*}\left(K \wedge_{E} B\right)
$$

is a G-Galois extension.

Proof. Let $C_{h}$ be the cofibre of $h: B \wedge_{E} B \longrightarrow \prod_{G} B$ in the category of $E$-module spectra. We know that $K \wedge C_{h} \simeq *$ and with the facts mentioned in Remark 7.1 we see that this is equivalent to $K \wedge_{E} C_{h} \simeq *$. The argument for the map $i: E \longrightarrow B^{h G}$ is similar. With these two facts at hand we can mimic the proof of Proposition 3.6 to obtain the result.

\section{Theorem 7.9.}

(a) For an odd prime $p$ and a finite group $G$, every $K(n)$-local $G$-Galois extension of $E$ is non-connected.

(b) For $p=2$ and $G$ a finite group possessing a cyclic quotient, every $K(n)$-local $G$-Galois extension of $E$ is non-connected.

Proof. From Lemma 7.8 we know that $\pi_{*}(K) \longrightarrow \pi_{*}\left(K \wedge_{E} B\right)$ is a $G$-Galois extension. As a Galois extension $B$ is dualizable in $\mathcal{D}_{E, K}$ and the finiteness discussion above ensures that it is in fact a retract of a finite cell- $E$-module spectrum. We can therefore transfer our proofs to the $K(n)$-local setting, to show that $\pi_{0}(K) \longrightarrow \pi_{0}\left(K \wedge_{E} B\right)$ splits and we can lift the corresponding idempotents to $\pi_{0}(E) \longrightarrow \pi_{0}(B)$.

\section{Acknowledgements}

This paper is dedicated to Doug Ravenel and Steve Wilson who have led algebraic topologists into brave new lands.

A. Baker was partially supported by a YFF Norwegian Research Council grant while visiting the University of Oslo. We would like to thank John Rognes, Haynes Miller and the referee for important comments on an early version, and Mark Hovey for allowing us to include material appearing in Section 7. We also thank Björn Schuster for sharing a stimulating bottle of wine in Oberwolfach. 


\section{References}

[A] V. Angeltveit, Topological Hochschild homology and cohomology of $A_{\infty}$ ring spectra, Geom. Topol. 12 (2008), 987-1032.

[BL] A. Baker and A. Lazarev, On the Adams Spectral Sequence for $R$ modules, Algebr. Geom. Topol. 1 (2001), 173-199.

[BR:1] A. Baker and B. Richter, On the $\Gamma$-cohomology of rings of numerical polynomials and $E_{\infty}$ structures on $K$-theory, Comment. Math. Helv. 80 (2005), 691-723.

[BR:2] A. Baker and B. Richter, Invertible modules for commutative $\mathbb{S}$-algebras with residue fields, manuscripta math. 118 (2005), 99-119.

[BR:3] A. Baker and B. Richter, Realizability of algebraic Galois extensions by strictly commutative ring spectra, Trans. Amer. Math. Soc. 359 (2007), 827-857.

[CHR] S. U. Chase, D. K. Harrison and A. Rosenberg, Galois theory and Galois cohomology of commutative rings, Mem. Amer. Math. Soc. 52 (1965), $15-33$.

[DeMI] F. DeMeyer and E. Ingraham, Separable Algebras over Commutative Rings, Lecture Notes in Mathematics 181, Springer-Verlag, New York, 1971.

[DH] E. S. Devinatz and M. J. Hopkins, Homotopy fixed point spectra for closed subgroups of the Morava stabilizer groups, Topology 43 (2004), $1-47$.

[E] D. Eisenbud, Commutative Algebra With a View Toward Algebraic Geometry, Graduate Texts in Mathematics, 150, Springer-Verlag, Berlin and New York, 1995.

[EKMM] A. Elmendorf, I. Kriz, M. Mandell and J. P. May, Rings, Modules, and Algebras in Stable Homotopy Theory, Mathematical Surveys and Monographs 47, AMS Bookstore, 1997.

[GH] P. G. Goerss and M. J. Hopkins, Moduli spaces of commutative ring spectra, in Structured Ring Spectra, London Math. Soc. Lecture Note Series 315, Cambridge Univ. Press, 2004, 151-200.

[GHMR] P. G. Goerss, H.-W. Henn, M. Mahowald and C. Rezk, A resolution of the $K(2)$-local sphere at the prime 3, Ann. of Math. 162 (2005), 777-822.

[Ho] M. Hovey, Morava E-theory of filtered colimits, Trans. Amer. Math. Soc. 360 (2008), 369-382.

[HS] M. Hovey and N. P. Strickland, Morava $K$-theories and localisation, Mem. Amer. Math. Soc. 139 (1999), no. 666.

[Re] C. Rezk, Notes on the Hopkins-Miller theorem, in Homotopy Theory Via Algebraic Geometry and Group Representations (Evanston, IL, 1997), Contemp. Math. 220, Amer. Math. Soc., Providence, RI, 1998, 313-366.

[RR] B. Richter and A. Robinson, Gamma-homology of group algebras and of polynomial algebras, Contemp. Math. 346 (2004), 453-461. 
[R] J. Rognes, Galois extensions of structured ring spectra, Mem. Amer. Math. Soc. 192 no. 898 (2008), 1-97.

[SVW] R. Schwänzl, R. M. Vogt and F. Waldhausen, Adjoining roots of unity to $E_{\infty}$ ring spectra in good cases - a remark, Contemp. Math. 239 (1999), 245-249.

[Sh] S. Shatz, Profinite Groups, Arithmetic and Geometry, Annals of Mathematics Studies 67, Princeton University Press, 1972.

[We] C. A. Weibel, An Introduction to Homological Algebra, Cambridge Studies in Advanced Mathematics 38, Cambridge University Press, Cambridge, 1994.

[Wü] S. Wüthrich, Infinitesimal thickenings of Morava $K$-theories, J. Pure Appl. Algebra 212 (2008), 99-121.

Andrew Baker a.baker@maths.gla.ac.uk http://www.maths.gla.ac.uk/ ajb Department of Mathematics, University of Glasgow, Glasgow G12 8QW, Scotland.

Birgit Richter richter@math.uni-hamburg.de http://www.math.uni-hamburg. de/home/richter/

Department Mathematik der Universität Hamburg, 20146 Hamburg, Germany. 